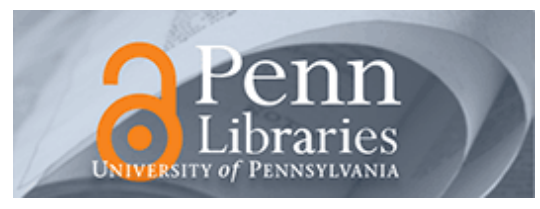

University of Pennsylvania ScholarlyCommons

\title{
DESIGN OF A MULTI-DIRECTIONAL VARIABLE STIFFNESS LEG FOR DYNAMIC RUNNING
}

\author{
Kevin C. Galloway \\ University of Pennsylvania, kcg@seas.upenn.edu \\ Jonathan E. Clark \\ Florida State University, clarkj@eng.fsu.edu \\ Daniel E. Koditschek \\ University of Pennsylvania, kod@seas.upenn.edu
}

Follow this and additional works at: https://repository.upenn.edu/ese_papers

Part of the Engineering Commons

\section{Recommended Citation}

Kevin C. Galloway, Jonathan E. Clark, and Daniel E. Koditschek, "DESIGN OF A MULTI-DIRECTIONAL VARIABLE STIFFNESS LEG FOR DYNAMIC RUNNING", . January 2007.

@inproceedings $\{$ Galloway.07, Author $=\{$ Galloway, K.C. and Clark, J.E. and Koditschek, D.E. $\}$, Title $=\{$ Design of a Multi-Directional Variable Stiffness Leg for Dynamic Runnings $\},$ BookTitle $=\{$ ASME Int. Mech. Eng. Congress and Exposition $\}$, Year $=\{2007\}\}$

This paper is posted at ScholarlyCommons. https://repository.upenn.edu/ese_papers/508

For more information, please contact repository@pobox.upenn.edu. 


\title{
DESIGN OF A MULTI-DIRECTIONAL VARIABLE STIFFNESS LEG FOR DYNAMIC RUNNING
}

\begin{abstract}
Recent developments in dynamic legged locomotion have focused on encoding a substantial component of leg intelligence into passive compliant mechanisms. One of the limitations of this approach is reduced adaptability: the final leg mechanism usually performs optimally for a small range of conditions (i.e. a certain robot weight, terrain, speed, gait, and so forth). For many situations in which a small locomotion system experiences a change in any of these conditions, it is desirable to have a variable stiffness leg to tune the natural frequency of the system for effective gait control. In this paper, we present an overview of variable stiffness leg spring designs, and introduce a new approach specifically for autonomous dynamic legged locomotion. We introduce a simple leg model that captures the spatial compliance of the tunable leg in three dimensions. Lastly, we present the design and manufacture of the multi-directional variable stiffness legs, and experimentally validate their correspondence to the proposed model.

\section{Disciplines}

Engineering

\section{Comments}

@inproceedings $\{$ Galloway.07, Author $=\{$ Galloway, K.C. and Clark, J.E. and Koditschek, D.E. $\}$, Title $=$ \{Design of a Multi-Directional Variable Stiffness Leg for Dynamic Runnings $\},$ BookTitle $=\{$ ASME Int. Mech. Eng. Congress and Exposition $\}$, Year $=\{2007\}\}$
\end{abstract}




\section{DESIGN OF A MULTI-DIRECTIONAL VARIABLE STIFFNESS LEG FOR DYNAMIC RUNNING}

\author{
Kevin C. Galloway * \\ Mechanical Engineering \\ and Applied Mechanics \\ University of Pennsylvania \\ Philadelphia, PA 19104 \\ Email: kcg@seas.upenn.edu
}

\author{
Jonathan E. Clark \\ Mechanical Engineering \\ Florida A\&M - Florida State University \\ Tallahasse, FL 32310
}

\author{
Daniel E. Koditschek \\ Electrical and Systems Engineering \\ University of Pennsylvania \\ Philadelphia, PA 19104
}

\begin{abstract}
Recent developments in dynamic legged locomotion have focused on encoding a substantial component of leg intelligence into passive compliant mechanisms. One of the limitations of this approach is reduced adaptability: the final leg mechanism usually performs optimally for a small range of conditions (i.e. a certain robot weight, terrain, speed, gait, and so forth). For many situations in which a small locomotion system experiences a change in any of these conditions, it is desirable to have a variable stiffness leg to tune the natural frequency of the system for effective gait control. In this paper, we present an overview of variable stiffness leg spring designs, and introduce a new approach specifically for autonomous dynamic legged locomotion. We introduce a simple leg model that captures the spatial compliance of the tunable leg in three dimensions. Lastly, we present the design and manufacture of the multi-directional variable stiffness legs, and experimentally validate their correspondence to the proposed model.
\end{abstract}

\section{INTRODUCTION}

Recently the design of variable stiffness compliant joints in robotic appendages has begun to receive increased attention. This has been motivated, in part, by the fact that biological systems can vary the stiffness of their limbs in real time to adapt to changes in the environment or expectant changes in the task [1],

\footnotetext{
*Address all correspondence to this author.
}

[2]. In fact, [1] has suggested that adjustable leg stiffness is necessary to close the performance gap between robots and animals.

Traditionally, stiffness control in mechanisms has been implemented at the motor level by adjusting the gains at individual joints. For dynamic tasks with significant impacts of unpredictable timing such as running, the inherent power limitations and bandwidth delays in motor control have led to the development and adoption of passive compliant legs. The use of actuators with mechanical spring elements rather than stiffness control of the actuated joints has the advantages of zero-lag (infinite bandwidth) and the possibility of energy storage and return.

Inspired by Raibert's work on simple dynamic hopping robots [3], mechanical leg springs have been incorporated into a number of running platforms including Scout [4], [5], Tekken [6], Whegs [7], [8], Sprawlita [9], and RHex [10]. RHex, a variant of which is the experimental platform utilized in this work, is one of the most successful autonomous running robots to date. It is the first autonomous dynamic legged locomotion system to passively exchange spring energy through natural body dynamics. It is also the fastest autonomous legged robot capable of operating on rough terrain.

Our previous work in building dynamic running legged robots has indicated that properly designed passive elements in the legs contribute in at least three ways to successful dynamic locomotion.

i. Leg springs act as low-pass filters on the impact forces from ground contact, which reduces the shock experienced by the robot's body, thereby significantly increasing the physical ro-
Copyright (c) 2007 by ASME 
bustness of the system.

ii. Springs act in concert with the rhythmically excited actuators providing a very high bandwidth energy exchange element - essentially a tuned harmonic system — increasing the efficiency of locomotion.

iii. Properly designed spring elements contribute to the overall stability of the robot against perturbative forces.

For example, both the Sprawl and RHex robots run in an extroceptively open loop manner. A simple feed-forward oscillatory activation scheme excites the legs, and it is the interaction of the passive mechanical system with the substrate that induces convergent forward locomotion. Even in the face of significant out-of-plane perturbations or rough terrain these robots run in a dynamically stable manner. Although the complexity of interaction of the dynamics and the distributed compliance in the legs has thus far precluded a clear analytical understanding of how to tune the legs for optimal stability, our empirical experience has shown that small changes in the magnitude or orientation of the spring elements has a dramatic effect on the stability of the resulting motion $[11,12]$.

Research suggests that variable compliance should increase adaptability, efficiency and robustness of autonomous dynamic runners [13], [14]. Recently, a few groups have begun integrating variable compliant springs into dynamic bipedal locomotion systems [14-16]. None of these systems, however, have, to our knowledge, thus far been able to empirically demonstrate the advantage of variable compliant legs for running robots.

On the other hand, the RHex compliant C-shaped leg has proven very successful [17], but offers only one effective stiffness. It is significant to note that the passive properties of these legs are not restricted to a single rotary or prismatic joint, but are spatial-with different effective stiffnesses in each direction. The motivation of this work is to extend the capabilities of an already proven dynamic legged locomotion system by adding variable compliant legs to improve gait control and efficiency over a range of forward speeds. In the process, we seek to better understand the role of distributed, spatial leg stiffness in stabilizing and propelling dynamic legged robots. The organization of the remainder of the paper is as follows: in Section II, we review existing variable compliant joint design approaches. Section III discusses the state-of-the-art in compliant leg design for our dynamical running robot, and proposes a new model to capture the spatial compliant properties of the limb. One significant advantage of this model is that it can be effectively utilized in the design of a variable passive compliant leg. In Section IV, we discuss the technique used to manufacture desired leg geometries, and in section $\mathrm{V}$ we empirically show how this model matches the stiffness ranges of the new legs. In Section VI, we close with a discussion of future research in the development in robots with variable passive compliant legs.

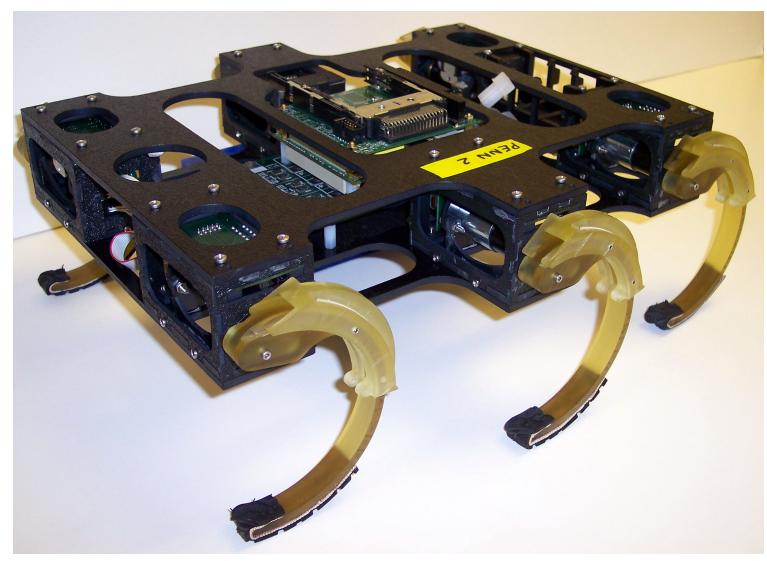

Figure 1. TUNABLE STIFFNESS LEGS ADAPTED TO EDUBOT, A RHEX INSPIRED DYNAMIC LEGGED LOCOMOTION ROBOT.

\section{VARIABLE PASSIVE COMPLIANT ACTUATORS}

Variable passive compliant actuators come in several configurations, the most common of which are: an antagonistic set-up of 2-non-linear springs, mechanical stiffness control, and structurally controlled stiffness.

Recently [16] and [14] have developed variable compliant joints that utilize an antagonistic set-up of two non-linear springs. In [16], the PPAM (Pleated Pneumatic Artificial Muscles) is a muscle-like actuator that uses a pair of opposing pleated membranes which contract longitudinally when pressurized with air. The actuation technology offers a favorable power to weight ratio; however, the difficulty of transporting pressurized air makes it an unlikely candidate for autonomous locomotion. In [14], the AMASC (Actuator with Mechanically Adjustable Series Compliance) shows more promise for integration into an autonomous dynamic legged locomotion system. Its construction places two non-linear springs in direct opposition to each other. The joint stiffness is tuned through software by adjusting two motor controlled inputs, which determine the spring set point and spring stiffness. A common problem among these types of systems is that they tend to be very complex and rely heavily on software control architectures and sensors to adjust or maintain stiffness. Furthermore, in antagonistic systems it is usually the case that joint compliance and torque control can not be decoupled.

The MACCEPA (Mechanically Adjustable Compliance and Controllable Equilibrium Position Actuator) is an example of a mechanical joint stiffness control mechanism [18]. Joint stiffness is controlled by two servo motors; one adjusts the angle of a lever arm which sets the equilibrium point, and the other pretensions the spring independently of the equilibrium position. The MACCEPA is a simple design and works well for controlled passive walking; however, the power and weight cost of supporting two motors to control a single joint stiffness makes it a difficult approach to implement on a RHex scale robot. 
The third common type of compliant actuation, known as structurally controlled stiffness, is a variable compliant method that changes the active structure of some elastic element such as a helical spring or a bending beam. Usually compliance is changed by adjusting the active length of a spring or the deflection point on a beam. Several groups have developed structurally controlled stiffness mechanisms [19-21], though none of them have been applied toward the development of autonomous dynamic legged locomotion systems.

A characteristic of each of the above variable stiffness mechanisms is that they have been designed to control an individual joint stiffness. The mechanism is effectively a revolute or prismatic joint, and two motor inputs are typically required to define or maintain joint stiffness. Thus, in reference to roles of springs outlined in Section I, the PPAM, AMASC, and MACCEPA fulfill (i) and (ii) but are ill equipped to provide (iii) for a small robot. Furthermore, for the open loop feed forward strategy of the RHex platform, these approaches would be very difficult to implement at the RHex scale and still offer the design flexibility and the stiffness and deflection range that this platform demands.

In this paper, our goal is to design a structurally controlled stiffness leg with properly tuned passive-mechanical properties in three dimensions that can adapt these properties for efficient running at a number of forward speeds, loads, or surface conditions. As a base point, our robot's current legs are about $12 \mathrm{~cm}$ long, weigh on the order of $60 \mathrm{~g}$, undergo loads of up to three times the robot's body weight, and can deflect in both the sagittal plane and lateral direction. Any variable stiffness leg design should preserve these characteristics and should allow for a significant range of mechanical stiffnesses to be achieved.

\section{STRUCTURALLY CONTROLLED STIFFNESS LEG MODEL}

As a base point for our variable-stiffness leg design, we chose the current passive compliant limbs of the RHex robot. RHex is a shoe-box size, compliant hexapod that has empirically demonstrated impressive performance on even the roughest terrains [22]. Its construction consists of a rigid body and six compliant legs that each have one independently actuated revolute degree of freedom [10]. There have been several iterations on the compliant leg design [17], with the intial leg built from a curved rod of delrin which was quickly abandoned for its inappropriate stiffness compliance and its fragility. The second major iteration was a 4-bar linkage design whose compliance was generated by the deformation of two fiberglass components on the internal 4bar linkage mechanism [23]. This planar mechanism was easier to model, and had better deflection properties, but still had robustness issues. The current leg design is a semi-circular shaped fiberglass beam. The curved shape of the leg aids in standing from rest and allows for the contact point to roll during stance.

Despite its success, little work has been conducted to un-
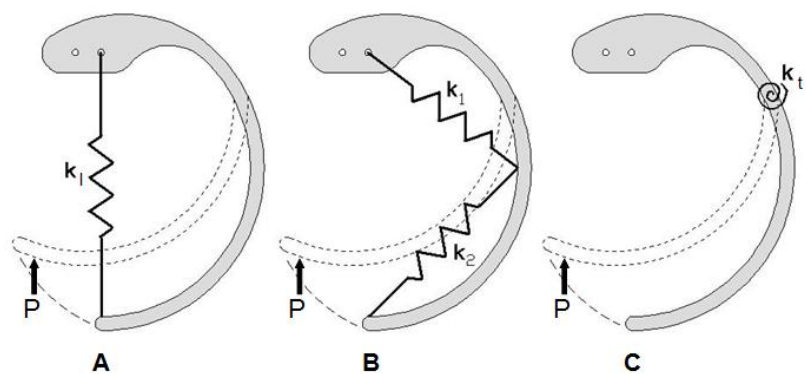

C

Figure 2. ILLUSTRATIONS OF THE DIFFERENT SPRING MODELS USED TO UNDERSTAND C-LEG COMPLIANCE UNDER LOAD, P. (A) RESPRESENTS THE LINEAR MODEL (B) REPRESENTS THE 2DIMENSIONAL MODEL (C) REPRESENTS THE PSEUDO-RIGID-BODY MODEL WHERE STIFFNESS IS CHARACTERSIZED BY A TORSIONAL SPRING.

derstand the C-leg's non-linear behavior under load. For the purposes of modeling, it has typically been simplified to a single linear spring even though under load the leg end clearly deflects in 2 dimensions (see Fig. 2A). In [23], it was modeled as a two degree of freedom system by two orthogonally placed linear springs (see Fig. 2B). Although, the two spring model captures the force-deflection behavior of the compliant leg, it is difficult to work with due to the number of parameters needed to specify the orientation and magnitude of the springs. We propose a new model to capture the spatial compliant properties of the leg using a combination of pseudo-rigid-body (PRB) model (see Fig. 2C) and standard beam bending theory. This model represents an implementation of the pseudo-rigid-body model for curved beams, where the leg stiffness is represented by a torsional spring at the effective center of rotation.

\section{Compliance in the Sagittal Plane}

In the pseudo-rigid-body model, flexible members are represented as rigid links connected via pin joints with torsional springs (see Fig. 3) [24]. This approach was chosen for two reasons. First, the path followed by the leg end, or toe, is nearly circular. Thus, representing the leg stiffness as a torsional spring best captures the large, curved deflections of the leg under load. Second, the PRB model offers design and time saving advantages. For example, it is significantly easier to estimate the leg stiffness for different configurations and dimensions using the PRB model than it is to update a solid model and constraints in a finite element program.

In this model, the initial curvature and the length of the leg link are related through the non-dimensionalized parameter

$$
k_{o}=\frac{l}{R_{i}}
$$




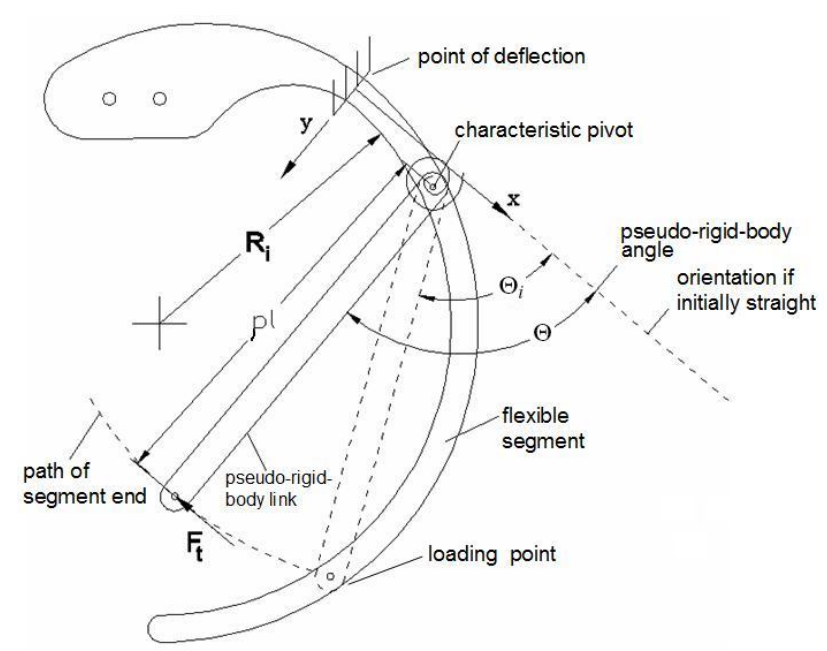

Figure 3. PSEUDO-RIGID-BODY MODEL APPLIED TO THE C-LEG. ADAPTED FROM [24]

where $l$ is the leg arc length measured along the centroidal axis of the leg from the point of deflection to the loading point, and $R_{i}$ is the initial radius of the curved beam. Figure 3 details the components of the PRB model where the characteristic radius factor, $\rho$, is used to determine the location of the the characteristic pivot and the length of the pseudo-rigid-body link. The PRB angle, $\Theta$, specifies the angle of the PRB link while, $\Theta_{i}$, defines the initial angle of the PRB link. Detailed explanations of the PRB model can be found in [24]; however, for the purposes of this paper we are primarily interested in the magnitude of the torsional spring constant, $K_{t}$, which is given by

$$
K_{t}=\rho K_{\Theta} \frac{E I_{s}}{l}
$$

where $K_{\Theta}$ is the stiffness coefficient, E is the Young's modulus, and $I_{S}$ is the moment of inertia in the sagittal plane. For initially straight beams $K_{\Theta}$ is a function of the angle at which the load is applied. For initially curved beams and $k_{o}$ values near 1.0 and higher, $K_{\Theta}$ is relatively constant for tangential and compressive beam loading. This means that $K_{\Theta}$ can be approximated from $k_{o}$. In the same way, for given $k_{o}$ values, $\rho$ can also be averaged for a range of loading conditions. These approximations have been captured in a simple look-up table in [25]. Therefore, E, $I_{s}, R_{i}$, and $l$ are all that is needed to approximate $K_{t}$ in the PRB model.

\section{Compliance in the Lateral Direction}

The C-leg also has compliance in the lateral direction or the direction normal to the sagittal plane. The leg stiffness in this direction, $K_{l}$, can be characterized by the standard cantilever beam

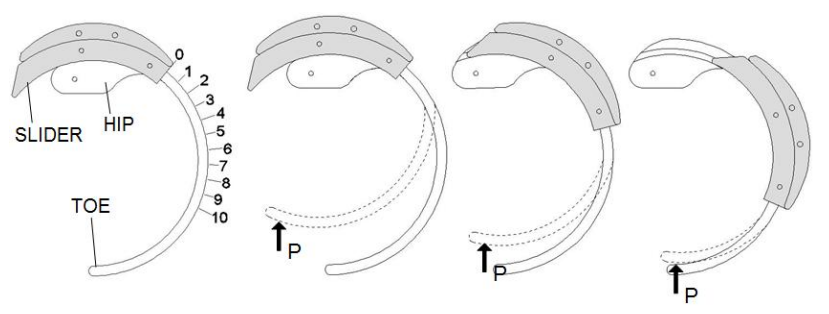

Figure 4. AN IMPLEMENTATION OF A STRUCTURALLY CONTROLLED STIFFNESS MECHANISM APPLIED TO A C-LEG.

bending equation

$$
K_{l}=3 \frac{E I_{l}}{L^{3}}
$$

where $\mathrm{L}$ is the linear distance from the point of deflection to the loading point, and $I_{l}$ is the moment of inertia in the lateral direction.

It is important to note that $K_{t}$ and $K_{l}$ can be independently specificed by changing the moment of inertia. This feature increases design flexibility and allows one to adjust spatial compliance in the lateral direction independent of the sagittal plane. Our model assumes that small deflections in the lateral direction causes a negligible deflection in the saggital plane, allowing us to consider the motions effectively decoupled.

\section{Structurally Controlled Stiffness C-leg}

To vary the leg stiffness for the next generation of hexapedal robots, a robust sliding mechanism, labeled as "slider" in Figure 4 , has been added. It is assumed that any portion of the leg that is covered by the slider is rigid, and the remaining exposed portion of the leg is compliant. In the sagittal plane, moving the slider changes the length of the PRB link and effectively shifts the location and magnitude of the torsional spring constant. The same result is true for stiffness in the lateral direction, in which the slider changes the value of $L$ in Eqn. 3. In Fig. 4, the slider can move continuously between the 0 and 10 markings where 0 is the most compliant configuration and 10 is the stiffest. Using the PRB model and the lateral stiffness equation, we can predictably design the tunable leg to operate within a range of stiffnesses as long as a portion of the slider is supported by the hip region. For example, if the slider moves past the 10th marker it loses support from the hip region and the leg will begin to deflect from both ends of the slider.

It is important to note that moving the slider will affect the stiffness in all directions in a coupled manner. Fortunately the intuition offered from the primary biomechanical running models - the Spring-Loaded Inverted Pendulum model [26] in the sagittal plane and Lateral Leg Spring model [27] — suggest that 


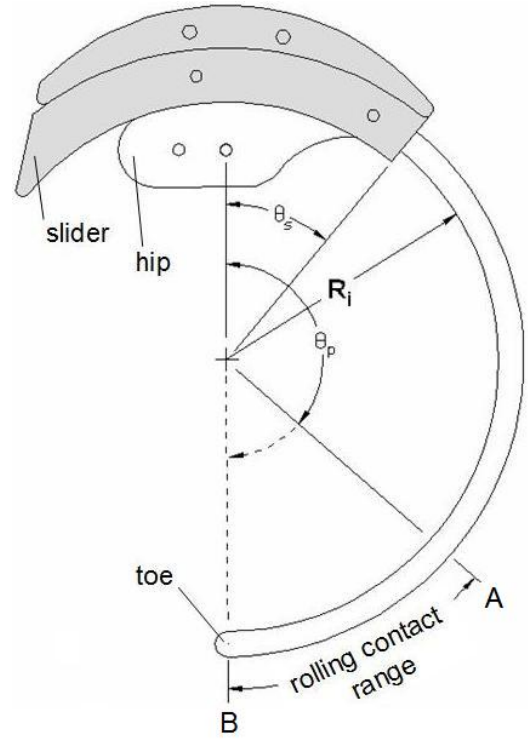

Figure 5. APPLICATION OF PRB-MODEL TO TUNABLE LEG WHERE LEG STIFFNESS CAN BE DEFINED BY THE SLIDER POSITION AND THE LOADING POINT.

the change in stiffness in each direction should increase with running frequency or robot load. Although the optimal nature of this coupling for a many-legged spatial robot has not yet been worked out in detail, our design couples these changes in the right trend.

\section{PRB Based Leg Model}

Thus far the PRB model has been presented with a single loading force where the loading point does not change. During operation of the robot, however, the loading point changes significantly. Generally, the leg touches down at around Point A (see Figure 5) and rolls through to about Point B during the stance phase. The value of $K_{t}$ decreases from A to B according to Eqn. 2 as the value of $l$ increases. Although calculating the effective stiffness using PRB-based model of bending is more complex than with a simple linear prismatic spring, there are two notable features about the $\mathrm{C}$-leg that make it difficult to reduce it to the simple spring model. First, there exists a coupled twodimensional compliant behavior in the sagittal plane. Second, as the leg rolls during the stance phase, the moving point of contact creates two behavoirs that can not be captured by a prismatic model. The first is that the stiffnesss of the leg decreases as the leg progress from touch down to lift off. The PRB model captures this behaviour as changes in $l$, but the linear model can not. The other non-linear spring behavior is that the rest length $l$ increases as the leg rolls through the stance phase. Our experience in designing and testing alternative legs is that failure to incorporate these behaviors leads to the design of legs with poor performance characteristics.

Even though stiffness varies along the length of the leg, it is not critical to determine the exact stiffness of the leg for each loading point since the robot will be optimized for different stiffness settings. In the design stage, it is more important to consider the range of stiffnesses, or relative stiffness of the leg. To calculate the range of stiffnesses for the C-leg presented in Figure 5, $k_{o}$ can also be represented as

$$
k_{o}=\theta_{p}-\theta_{s}
$$

where in radians $\theta_{p}$ specifies the loading point, and $\theta_{s}$ species the angular position of the slider or point of deflection. Thus to design a C-leg for a range of stiffnesses in the sagittal plane there are several design variables in the model that can be tuned including the range of $\theta_{s}, E, I_{s}$, and $R_{i}$.

\section{DESIGN AND MANUFACTURING}

Early in the development of the structurally controlled stiffness C-leg a number of target specifications were generated. First, the leg should have a maximal radial length of $12 \mathrm{~cm}$. Second, based off of previous leg designs, the lateral stiffness should also be at least $300 \mathrm{~N} / \mathrm{m}$ and the torsional stiffness in the sagittal plane should vary from about 2.75 to $5.5 \mathrm{Nm} / \mathrm{rad}$ so that the natural frequency of the legs matches the range of driving frequencies. Furthermore, during dynamic loading each leg should be able to support the weight of the robot, and not exceed the yield strength of the material.

Thus for the manufacture of the variable stiffness C-leg, it was important to select a method which would allow considerable design flexibility to test various materials and shapes quickly and economically, and one that offers the option of integrating parts to save volume and weight. These criteria were satisfied through Shape Deposition Manufacturing (SDM), a solid freeform fabrication process which systemically combines material deposition with material removal processes. The general SDM design principles and techniques are covered in detail in [28], and have been applied to robotics [9, 29-31]. SDM offers several advantages over traditional prototyping methods. Some of these include the ease of embedding components (i.e. actuators and electronics), the flexibility of combining dissimilar materials to create complex compliant mechanisms, creating whole parts in a layered fashion, and eliminating custom tooling [31]. For the fabrication of the tunable leg designs, the SDM process offers the advantage of adjusting the leg shape and design variables noted earlier. The overall leg stiffness can be adjusted by choosing an epoxy (see Table 1) from a family of materials (Innovative Polymers, Inc., Saint Johns, MI) of different Young's moduli, E, by changing the moment of inertia, I, or by changing the length of the slider. For example, the tunable legs used for 
Table 1. MATERIAL PROPERTIES

\begin{tabular}{cccc}
\hline Epoxy & $\mathrm{E}(M P a)$ & $S_{y}(M P a)$ & $\frac{S_{y}}{E} \times 1000$ \\
\hline TP-4000 & 690 & 21 & 30 \\
TP-4004 & 793 & 35 & 44 \\
TP-4007 & 2240 & 104 & 46 \\
\hline
\end{tabular}
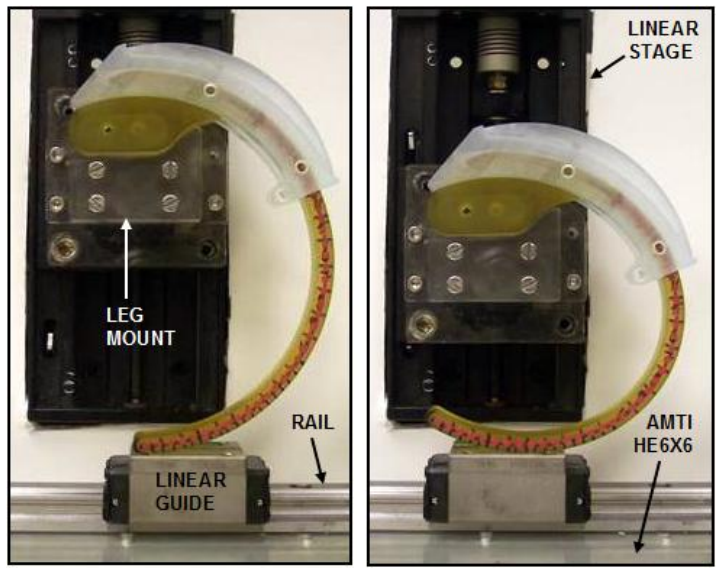

Figure 6. RELAXED AND COMPRESSED IMAGES OF A C-LEG IN THE EXPERIMENTAL SET-UP

testing are fabricated from TP-4004 which offers a relatively low Young's modulus with a favorable strength-to-modulus ratio. It is important to note that a high ratio will permit larger leg deflections before fracture and increases the design flexibility to create structures for a range of moments of inertia.

\section{MEASURING LEG STIFFNESS}

The $K_{t}$ for a shape deposition manufactured C-leg was collected at each of the slider positions shown in Figure 6. The leg was mounted to a Micos linear stage for ease of repeatability and the deflection of the leg against an AMTI HE6x6 force plate was visually captured. The linear stage has a resolution of one micrometer and is capable of traveling $80 \mathrm{~mm}$ at rates as high as $14 \mathrm{~mm} / \mathrm{s}$. The AMTI HE6x6 is a six axis force plate capable of measuring loads as large as 16 pounds at $200 \mathrm{hz}$ with 12 -bit resolution. A linear guide and rail were mounted to the force plate to prevent stick-slip behavoir during leg loading. Ten force measurements were collected with the linear stage pressing the leg into the force plate at $14 \mathrm{~mm} / \mathrm{s}$. The $K_{t}$ was obtained by marking evenly spaced colored dots along the centroidal axis of the leg. An image capture system was created to compare the relaxed and compressed images to determine the loading point, point of deflection, the characteristic pivot, the arc length, $l$, and the value

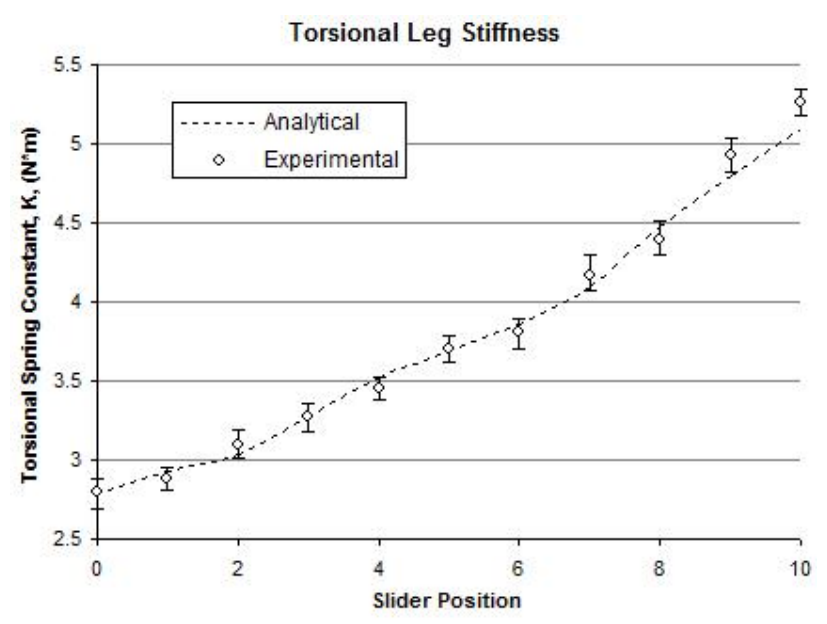

Figure 7. EXPERIMENTAL VALIDATION OF THE PRB MODEL FOR ESTIMATING TORSIONAL SPRING CONSTANT.

of the PRB-angle, $\Theta-\Theta_{i}$.

The analytical $K_{t}$ was calculated by inputting the specified material properties, and $l$ into equation (2). The value for $K_{\Theta}$ and $\rho$ were determined from the look-up table in [25].

The experimental $K_{t}$ was calculated by first measuring the resultant torque, $T_{R}$, about the characterstic pivot using the force data and the horizontal and vertical distances measured from the characteristic pivot to the loading point. The resultant torque along with the the PRB-angle, $\Theta-\Theta_{i}$, were then applied to the torsional spring equation below to determine the experimental torsional spring constant.

$$
K_{t}=\frac{T_{R}}{\Theta-\Theta_{i}}
$$

The stiffness in the lateral direction was determined by using the same force plate and linear stage. The toe was deflected in the lateral direction by pushing it into an obstruction rigidly anchored to the force plate. This experiment was repeated ten times for each even numbered slider position. A force-deflection graph was generated with the data, and a linear curve fit was applied to each experiment for a given slider position. The slopes of the linear curves were averaged to determine the average lateral leg stiffness for each slider position.

\section{Results}

For the sagittal plane stiffness, we found that the PRB model captured the behaviors of the leg under load reasonably well for a range of slider positions (see Figure 7). The error bars in Figure 7 reflect the range of torsional stiffness values obtained during testing. For each slider position the error between the analytical and 


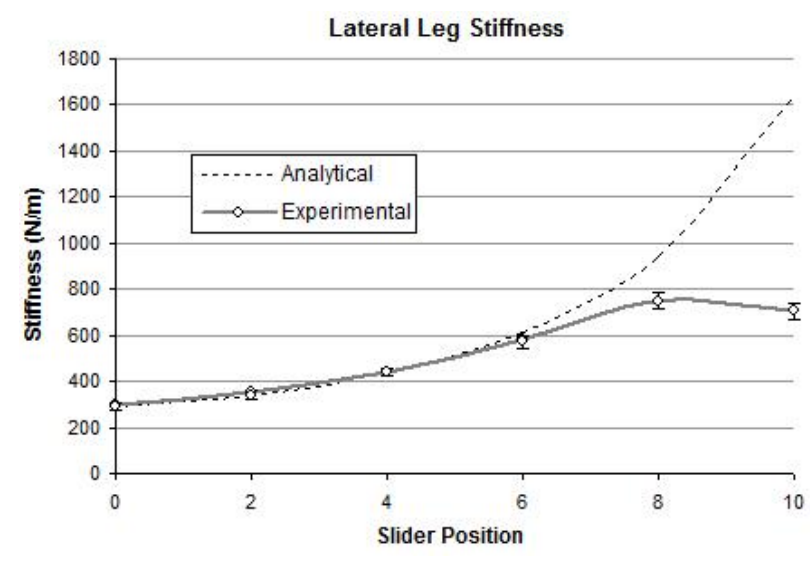

Figure 8. EXPERIMENTAL VALIDATION OF THE CANTILEVER BEAM BENDING MODEL FOR ESTIMATING LATERAL LEG STIFFNESS

average experimental torsional stiffness measurements was less then $3 \%$. For slider positions $0-8$, the analytical results fall within the error bars, however this not the case for positions 9 and 10 . This deviation can be attributed to deflection at the hip end of the slider. As mentioned earlier, as the slider moves to higher settings it is supported less and less by the hip region. For example, at slider position 10, there are noticeable deflections at both ends of the slider. Since this behavoir is not accounted for in the PRB model, it introduces an additional source of error.

Deflection at the hip end of the slider is even more apparent and occurs earlier in the lateral stiffness experimental results (see Figure 8). It is clear that the deviation between the analytical and experimental results begins near slider position 6 . As the slider moves to higher settings, deflections occur from both ends of the slider and the cantilever beam model is no longer valid.

In our model, we have assumed that the lateral motions and forces are decoupled. In reality there are radial and tangential forces generated when the leg is displaced laterally. Experiments using the force plate, however, indicate that for hip deflections in the lateral direction, the ratio of sagittal to lateral forces at the toe is less than $15 \%$ for all slider positions.

Structural controlled stiffness of the C-leg has its limitations, both in terms of directional coupling, and in the accuracy of the assumed linear model. It should be noted that the PRB model estimated that the torsional spring constant would increase by about $180 \%$ (from 2.8 to $5.1 \mathrm{~N}^{*} \mathrm{~m}$ ) at the stiffest setting and the experimental results showed almost a $190 \%$ increase. In the lateral direction, the stiffness increased by $270 \%$ (from 290 to 750 $\mathrm{N} / \mathrm{m}$ ), which is considerably lower than predicted. With a redesign, including a longer slider and slight adjustments to the geometry we are confident that the desired 2-3x torsional stiffness change can be achieved.

\section{CONCLUSION AND FUTURE WORK}

Biological studies and on-going robotic research suggest that variable stiffness legs for dynamic locomotion are a key control parameter in responding to changes in running gait, payload and terrain. In this paper, we have presented the design and construction of a structurally controlled stiffness limb which utilizes a single degree of freedom mechanism to control the stiffness properties of the leg in three dimensions. This design has grown out of a new model that captures the spatial compliant properties of the best current leg design for RHex. Furthermore, we have shown the correspondence between our simple model and the 3D passive properties of a new adjustable compliance leg.

With these new tunable legs we are now able to test the hypothesis that variable stiffness legs can substantially improve the performance of dynamic running systems. Once the behavioral advantages of variable stiffness legs have been emperically demonstrated, the next phase will be to leverage the capabilities of SDM through the integration of an actuator and sensors directly into the structure of the leg to precisely move and measure the position of the slider and deflection of the leg. With a proper communication and control stratagy these integrated 'smart' legs will be capable of run-time adaptations to changing environmental conditions, moving us one step closer to truely agile dynamic robots.

More generally we seek to understand how to properly design the whole-body passive dyanmics of a running system. Understanding how directional coupling of the compliance in the Cleg works provides a framework for further experimentation and modeling efforts to determine how the passive stiffness in future legs should be designed for optimal stability and efficiency in a running robot.

\section{ACKNOWLEDGMENT}

We would like to thank Dr. Pei-Chun Lin for his insightful comments, and Sam Russem for his help testing and fabricating legs. Jonathan Clark was supported by the IC Postdoctoral Fellow Program under grant number HM158204-1-2030. This work was also partially supported by the NSF FIBR grant \#0425878.

\section{REFERENCES}

[1] Ferris, D., Louie, M., and Farley, C., 1998. "Running in the real world: Adjusting leg stiffness for different surfaces". In Proceedings of the Royal Society London, Vol. 265, pp. 989-993.

[2] McMahon, T. A., 1984. "Mechanics of locomotion". International Journal of Robotics Research, 3(2), pp. 4-28.

[3] Raibert, M. H., 1986. Legged robots that balance. MIT Press series in artificial intelligence. MIT Press, Cambridge, Mass. 
[4] Papdopoulos, D., and Buehler, M., 2000. "Stable running in a quadruped robot with compliant legs". In Proc. of the IEEE International Conference on Robotics and Automation, pp. 444-449.

[5] Smith, J. A., and Poulakakis, I., 2004. "Rotary gallop in the untethered quadrupedal robot scout ii". In Proc. of the IEEE/RSJ International Conference on Intelligent Robots and Systems, Vol. 3, pp. 2556-2561.

[6] Fukuoka, Y., Kimuar, H., and Cohen, A. H., 2003. "Adaptive dynamics walking of a quadruped robot on irregular terrain based on biological concepts". International Journal of Robotics Research, 22(3-4), pp. 187-202.

[7] Lambrecht, B. G. A., Horchler, A. D., and Quinn, R. D., 2005. "A small, insect-inspired robot that runs and jumps". Proc. of the IEEE International Confer-ence on Robotics and Automation, pp. 1252-1257.

[8] Quinn, R. D., Nelson, G. M., Bachmann, R. J., Kingsley, D. A., Offi, J., and Ritzmann, R. E., 2001. "Insect designs for improved robot mobility". In Proceedings of CLAWAR 2001, Karlsruhe, Germany, D. Berns, ed., pp. 69-76.

[9] Cham, J. G., 2002. "On stability and performance in openloop running". PhD thesis, Stanford University.

[10] Saranli, U., Buehler, M., and Koditschek, D. E., 2001. "Rhex: A simple and highly mobile hexapod robot". International Journal of Robotics Research, 20(7), pp. 616-631.

[11] Clark, J. E., and Cutkosky, M. R., 2006. "The effect of leg specialization in a biomimetic hexapedal running robot". Journal of Dynamic Systems, Measurement, and Control, 128, pp. 26-35.

[12] Burden, S., Clark, J. E., Weingarten, J .and Komsouglu, H., and Koditschek, D. E., 2007. "Heterogeneous leg stiffness and roll in dynamic running". In IEEE - International Conference of Robotics and Automation.

[13] Alexander, R. M., 1990. "Three uses for springs in legged locomotion". International Journal of Robotics Research, 9(2), pp. 53-61.

[14] Hurst, W., C. J. E., and Rizzi, A., A., 2004. "An actuator with physically variable stiffness for highly dynamic legged locomotion". In Proceedings - IEEE International Conference on Robotics and Automation, pp. 4662-4667.

[15] Daerden, F., and Lefeber, D., 2001. "The concept and design of pleated pneumatic artificial muscles". International Journal of Fluid Power, 2(3), pp. 41-50.

[16] Van Ham, R., Van Damme, M., Vanderborght, B., Verrelst, B., and Lefeber, D., 2006. "Maccepa: The mechanically adjustable compliance and controllable equilibrium position actuator". In Proceedings of CLAWAR, pp. 196-203.

[17] Moore, E. Z., 2002. "Leg design and stair climbing control for the RHex robotic hexapod". Master's thesis, McGill University, Jan.

[18] Beyl, P., Vanderborght, B., Van Ham, R., Van Damme, M., Versluys, R., and Lefeber, D., 2006. "Compliant actuation in new robotic applications". In NCTAM06 - 7th National Congress on Theoretical and Applied Mechanics.

[19] Morita, T., and Sugano, S. "Design and development of a new robotic joint using a mechanical impedance adjuster". In IEEE International Conference on Robotics and Automation, Vol. 3.

[20] Hollander, K., Sugar, T., and Herring, D., 2005. "Adjustable robotic tendon using a 'jack spring". In Proceedings of the ASME Design Engineering Technical Conference.

[21] Tabata, O., Konishi, S., Cusin, P., Ito, Y., Kawai, F., Hirai, S., and Kawamura, S., 1999. "Microfabricated tunable stiffness device". Proc. of the 13th Annual Int. Conf. On Micro Electro Mechanical Systems.

[22] Weingarten, J., Groff, R., and Koditschek, D., 2004. "A framework for the coordination of legged robot gaits". In IEEE International Conference of Robotics, Automation and Mechatronics, Singapore.

[23] Lin, P.-C., 2005. "Proprioceptive sensing for a legged robot". PhD thesis, University of Michigan.

[24] Howell, L. L., 2001. Compliant Mechanisms. Wiley, New York.

[25] Howell, L. L., and Midha, A., 1996. "Parametric deflection approximations for initially curved, large-deflection beams in compliant mechanisms". In Proceedings of the ASME Design Engineering Technical Conference.

[26] Blickhan, R., and Full, R. J., 1993. "Similarity in multilegged locomotion: Bounding like a monopod". Journal of Comparative Physiology, 173(5), pp. 509-517.

[27] Schmitt, J., Garcia, M., Razo, R., Holmes, P., and Full, R., 2002. "Dynamics and stability of legged locomotion in the horizontal plane: a test case using insects.". Biological Cybernetics, 86(5), pp. 343-53.

[28] Merz, R., B., P. F., Ramaswami, K., Terk, M., and Weiss, L. E., 1994. "Shape deposition manufacturing". In Proceedings of the Solid Freeform Fabrication Symposium.

[29] Cham, J. G., Bailey, S. A., and Cutkosky, M. R., 2000. "Robust dynamic locomotion through feedforward-preflex interaction". In ASME IMECE Proceedings.

[30] Chitta, S., Karabas, M., Galloway, K., and Kumar, V., 2006. "Robotrikke: Design, modeling and experimentation with a robotic trikke". In Proceedings of the ASME Design Engineering Technical Conference.

[31] Dollar, A., and Howe, R., 2005. "Design and evaluation of a robust compliant grasper using shape deposition manufacturing". In Proceedings of the ASME Design Engineering Technical Conference. 